Original Research Paper

\title{
Performance Analysis of Threshold Based Compressive Sensing Algorithm in Wireless Sensor Network
}

\author{
Parnasree Chakraborty and C. Tharini \\ Department of Electronics and Communication Engineering, B.S.A. University, Chennai, 48. India
}

Article history

Received: $12-5-2015$

Revised: 24-01-2017

Accepted: 01-02-2017

Corresponding Author:

Parnasree Chakraborty

Department of Electronics and

Communication Engineering,

B.S.A. University, Chennai, 48.

India

Email: prernasree@bsauniv.ac.in

\begin{abstract}
Wireless Sensor Networks (WSN) are comprised of spatially distributed sensor nodes, where each node contains sensors, processors and transceivers for communicating data. Regardless of the application in which the sensor network is serving, the data generated in the network eventually must be delivered to the sink. However the limited network bandwidth, frequent node/link failure along with the unreliable communication medium poses great challenges for node to node communication in WSN. Hence, energy efficient data compression algorithms are necessary for sensor nodes as they enhance the transmission efficiency in WSN. Compressive sensing is a new compression algorithm in which the input signal is converted into sparse signal and the sparse signal is further converted into a signal of reduded dimension than original signal. The dimensionality reduction improves the transmission efficiency. This new concept is recently applied in WSN, however suitable threshold selection to sparsify the one dimensional sensor reading and suitable sparifying basis for image input data are not considered in literature. Hence, in this paper analysis of compressive sensing algorithm with a suitable threshold selection is performed in order to increase the level of sparsity for one dimensional data and a suitable sparsifying basis selection is performed for image data. Results indicate that compressive sensing with suitable threshold selection improves transmission and bandwidth efficiency in case of low correlated one dimensional sensor data and a suitable basis improves the quality of transmission for image sensor data and hence the overall lifetime of sensor network can be increased.
\end{abstract}

Keywords: Compressive Sensing, Threshold, Sparsity, WSN

\section{Introduction}

Wireless sensor network has wide range of applications, like medical, defense, transport and even our day to day life. A wireless node consists of sensors to collect the data, a processor and transceiver. This whole set up is supported by a battery which has limited life. Energy saving methodologies with different algorithms is needed to save the power in WSN as energy is limited in sensor node. The processes of CS aggregation in WSN are given in (Yang et al., 2013). A brief literature review is provided in related work section. Next Section deals with threshold based CS encoding method for one dimensional data. For image input data the suitable basis selection is analyzed. Different recovery methods for CS algorithm is dicussed in (Candès and Tao, 2006; Candès and Romberg, 2005). Last Section includes the conclusion.
The main idea of this paper is to exploit the nature of data correlation in WSN environment and reduce the number of data transmission. The proposed threshold based CS algorithm is applied to one dimensional signal and a suitable sparsifying basis is chosen for image input data. First computation of the correlation coefficient of the sensed one dimensional data is done and based on mean correlation coefficient a suitable threshold is chosen in order to increase the sparsity. This kind of analysis is not done in previous work to the best of our knowledge and hence the proposed work helps in reducing the number of data transmission in WSN.

\section{Related Work}

Generally data compression schemes for WSNs should be lightweight and the computational requirements of the algorithms should be low for 
efficient operation due to WSNs constraints in terms of hardware, energy, processing and memory. The most important issues and questions that have to be addressed in a wireless sensor network is proposed in (Karl and Willing, 2007). Many algorithms for data compression for WSN are found in literature. The modulation scaling for eneergy efficient network is proposed in (Schurgers et al., 2001). Some of other work shows adaptive modulation scaling which improves energy efficiency of WSN discussed in (Yang et al., 2005). Lightweight Data Compression in Wireless Sensor Networks using Huffman Coding proposed in (Medeiros and Maciel, 2014) uses Huffman algorithm for data reduction. A two-stage DPCM scheme for wireless sensor networks in (Luo et al., 2005) consists of temporal and spatial stages that compress data by making predictions based on samples from the past and helping sensors. A sensor node LZW (S-LZW) is designed specifically for resource constrained sensor nodes in (Marcelloni and Vecchio, 2008), Lossless Entropy Compression in (Marcelloni, 2009) exploits the high correlation that typically exists between consecutive samples collected by a sensor node but LEC algorithm achieves compression ratios of the order of $70 \%$, Median-Predictor-based Data Compression (MPDC) in (Kolo and Shanmugam, 2012) which performs compression losslessly using two code options and is suitable for both real-time and delaytolerant transmission.

The energy-model-based optimal communication systems design for wireless sensor networks are proposed in ( $\mathrm{Li}$ et al., 2012). While most of the literature focuses either on data compression algorithms or performance analysis of compression schemes for energy efficiency, still analysis is required about performance of compression algorithms for different type of input data. This work aims to initiate work in this direction and aims to analyze compressive sensing algorithm with and without threshold selection for one dimensional input data and also selection of suitable basis function for two dimensional image.

\section{Compressive Sensing Based Encoding for one Dimensional Data}

Compressive Sensing is a signal processing technique used in almost all the field of communication engineering for efficiently acquiring and reconstructing a signal, by finding solutions to underdetermined linear systems. In (Candès and Wakin, 2008), authors have discussed that according to CS theory, one can recover certain signals and images from far fewer samples or measurements than traditional methods use. Actually the CS algorithm works beyond the normal sampling theorem used in practice discussed in (Luke, 1999). To make this possible, CS relies on two principles: Sparsity, which pertains to the signals of interest and incoherence, which pertains to the sensing modality.
Sparsity (Candès and Wakin, 2008) expresses the idea that the "information rate" of a continuous time signal may be much smaller than suggested by its bandwidth, or that a discrete-time signal depends on a number of degrees of freedom which is comparably much smaller than its (finite) length. More precisely, CS exploits the fact that many natural signals are sparse or compressible in the sense that they have concise representations when expressed in the proper basis $\psi$.

Incoherence extends the duality between time and frequency and expresses the idea that objects having a sparse representation in $\psi$ must be spread out in the domain in which they are acquired, just as a Dirac or a spike in the time domain is spread out in the frequency domain. Put differently, incoherence says that unlike the signal of interest, the sampling/sensing waveforms have an extremely dense representation in $\psi$.

In CS method, the collected signals or images are changed into compressed sparse signals with any one of available kernel. Sparse signal are very few in number and has full structure and complete meaningful information which are necessary to reconstruct the original signal or image. Equation 1 describes generation of the sparse signal (s):

$s=T x$

In Equation 1, Where, $(x)$ is the input signal of size $(n \times 1),(T)$ is the Transform matrix or kernel of size $(n \times n)$ and $(s)$ is the sparse representation of size $(n \times 1)$. After sparse generation, measurement matrix $(M)$ of size $(m \times n)$ is used to compress the sparse representation ' $s$ ' of input image and compressed sparse output ' $y$ ' of size $(m \times 1)$ is generated. Here $m<<n$ and hence the resultant signal y is of reduced size $(m \times 1)$. The compressed sparse output $(y)$ is given in Equation 2:

$y=M s$

For the purpose of analysis the input signals are considered based on the correlation coefficient as highly correlated and low correlated data. Table 2 and 4 describe the thresholding effect on different correlated data.

The proposed threshold based CS algorithm for one dimensional signal computes the correlation coefficient of the sensed data which is used as a metric to decide the measurement matrix of the CS encoder. Further an upper threshold and a lower threshold based on Gaussian distribution is calculated to enforce the sparsity in transformed data. It is observed that the measurement matrix selection based on correlation characteristics of the data greatly improves the quality of reconstruction. Also thresholding technique increased the sparsity of the transformed data and hence the compression ratio is also improved. In order to enforce the sparsity level, thresholding is performed based on the following rule: 
- $\quad$ Find the mean of input data set by adding all the data points and dividing by the number of data points

- Subtract the mean from each data point and square the result

- For the given input data the mean square error, signal to noise ratio are calculated

- $\quad$ Take the square root of that mean to get the standard deviation

- Multiply the standard deviation by 3

- Add the mean of the original data set to the result. This is the upper control limit

- Subtract the result of Step 1 from the mean of the original data set to get the lower control limit

The performance of the proposed algorithm is measured by considering the input as temperature sensor data (200 nos. of sample). The Signal to Noise Ratio (SNR) and Mean Square Error (MSE) values are calculated for a temperature input of size 200 samples with mean correlation coefficient of 0.6565 which is shown below in the Table 1.

Table 1. Performance analysis of CS without thresholding for temperature samples of mean correlation coefficient 0.6565

\begin{tabular}{llll}
\hline $\begin{array}{l}\text { Temperature } \\
\text { data size }\end{array}$ & $\begin{array}{l}\text { Compressed } \\
\text { data } \text { size }\end{array}$ & $\begin{array}{l}\text { Mean Square } \\
\text { Error }(\mathrm{mse})\end{array}$ & $\begin{array}{l}\text { SNR } \\
(\mathrm{dB})\end{array}$ \\
\hline 200 & 100 & 0.0013 & 52.2617 \\
200 & 67 & 0.0049 & 46.6439 \\
200 & 50 & 0.0179 & 40.9886 \\
\hline
\end{tabular}

Table 2. Performance analysis of threshold based CS for temperature samples of mean Correlation coefficient 0.6565

\begin{tabular}{lllc}
\hline $\begin{array}{l}\text { Temperature } \\
\text { data size }\end{array}$ & $\begin{array}{l}\text { Compressed } \\
\text { data size }\end{array}$ & $\begin{array}{l}\text { Mean Square } \\
\text { Error }(\mathrm{mse})\end{array}$ & $\begin{array}{l}\text { SNR } \\
(\mathrm{dB})\end{array}$ \\
\hline 200 & 100 & 0.0016 & 51.4694 \\
200 & 67 & 0.0076 & 44.6987 \\
200 & 50 & 0.0502 & 36.5154 \\
\hline
\end{tabular}

Table 3.Performance analysis of CS without thresholding for temperature samples of mean Correlation coefficient 0.3076

\begin{tabular}{llll}
\hline $\begin{array}{l}\text { Temperature } \\
\text { data size }\end{array}$ & $\begin{array}{l}\text { Compressed } \\
\text { data size }\end{array}$ & $\begin{array}{l}\text { Mean Square } \\
\text { Error }(\mathrm{mse})\end{array}$ & $\begin{array}{l}\text { SNR } \\
(\mathrm{dB})\end{array}$ \\
\hline 200 & 100 & 0.6126 & 25.6501 \\
200 & 67 & 0.7960 & 24.5130 \\
200 & 50 & 1.0496 & 23.3115 \\
\hline
\end{tabular}

Table 4. Performance analysis of threshold based CS for temperature samples of mean Correlation coefficient 0.3076

\begin{tabular}{llll}
\hline $\begin{array}{l}\text { Temperature } \\
\text { data size }\end{array}$ & $\begin{array}{l}\text { Compressed } \\
\text { data size }\end{array}$ & $\begin{array}{l}\text { Mean Square } \\
\text { Error (mse) }\end{array}$ & SNR \\
\hline 200 & 100 & 0.5950 & 25.9278 \\
200 & 67 & 0.6778 & 25.2110 \\
200 & 50 & 0.9139 & 23.9128 \\
\hline
\end{tabular}

Table 5. MSE and PSNR (in dB) values in DCT basis with a input image dimension $32 \times 32$

\begin{tabular}{lll}
\hline $\begin{array}{l}\text { No. of samples } \\
\text { transmitted }\end{array}$ & MSE & PSNR $(\mathrm{dB})$ \\
\hline 700 & 0.2726 & 40.9535 \\
600 & 0.6835 & 38.9571 \\
500 & 0.8076 & 38.5948 \\
400 & 1.2609 & 37.6274 \\
300 & 2.1500 & 36.4658 \\
\hline
\end{tabular}

Table 6. MSE and PSNR (in $\mathrm{dB}$ ) values in DWT basis

\begin{tabular}{lll}
\hline $\begin{array}{l}\text { No. of samples } \\
\text { transmitted }\end{array}$ & MSE & PSNR (dB) \\
\hline 700 & 0.1896 & 41.74 \\
600 & 0.2020 & 41.6036 \\
500 & 0.2079 & 41.5147 \\
400 & 0.2489 & 41.1505 \\
300 & 0.4243 & 39.9922 \\
\hline
\end{tabular}

Table 1 Performance analysis of CS without thresholding for temperature samples of mean correlation coefficient 0.656 .

Table 2 shows performance analysis of threshold based CS for same set of input data.

The SNR comparison between basic CS and threshold based CS for highly correlated temperature data is shown in Fig. 1 and MSE comparison between basic CS and threshold based CS for highly correlated temperature data is shown in Fig. 2.

The similar analysis is done for another set of temperature data of mean correlation coefficient 0.3076 which is shown in Table 3 and 4.

The SNR comparison between basic CS and threshold based CS for temperature data of low correlation coefficient is shown in Fig. 3 and MSE comparison between basic CS and threshold based CS for highly correlated temperature data is shown in Fig. 4.

From the obtained result it is clear that thresholding technique gives better SNR and low MSE when input data are highly uncorrelated.

\section{Performance Analysis of Compressive Sensing Algorithm on Two Dimensional Signals}

Comparison of two different sparsifying basis is performed for image input signal of size $32 \times 32$. DWT and DCT basis are used for comparison. When DWT is used as sparsifying basis, the sparsity increases which results better reconstruction quality.

The input image is shown in Fig. 7. Table 5 shows the MSE and PSNR (in $\mathrm{dB}$ ) values in DCT basis with a input image dimension $32 \times 32$. Table 6 shows MSE and PSNR (in $\mathrm{dB}$ ) values in DWT basis. The concept of wavelet transform is well explained in (Mallat, 2008). 


\section{Recovery of Original Image}

In order to obtain original image, it is required to find the optimum solution for the Equation 2. Among many reconstruction techniques of $\mathrm{CS}$, Basis pursuit method is simple and hence for CS reconstruction it is used in this work. Basis Pursuit (BP) (Candès and Romberg, 2007; Candès and Tao, 2006; Candès and Romberg, 2005) is the one in which adopts either simplex iteration method or primal dual method. Reconstruction using basis pursuit method requires solving of underdetermined linear equations. This method is computationally complex and requires convex optimization algorithm. It uses a very few non-zero coefficients to reconstruct the signal. Basis Pursuit method involves $l_{1}$ norm optimization (Nesterov, 2004) to solve the underdetermined system of simultaneous linear equation of the form similar to Equation 2.

Figure 5. Shows the comparison of PSNR (in dB) in DCT and DWT basis and Fig. 6 shows about the mean square error(mse) in DCT and DWT basis. Figure 7 and 8 are reconstructed images using BP. Figure 7 is the reconstructed image generated using DCT transform. Figure 8 is the reconstructed image generated using DWT transform. Results indicate DWT provides less MSE and high PSNR making it suitable for images. The values of MSE and PSNR in $\mathrm{dB}$ are shown in Tables in previous section.

Result of this section was used to analyze a suitable sparsifying basis for the image input. From the result it is clear that DWT basis is better compared to DCT basis.

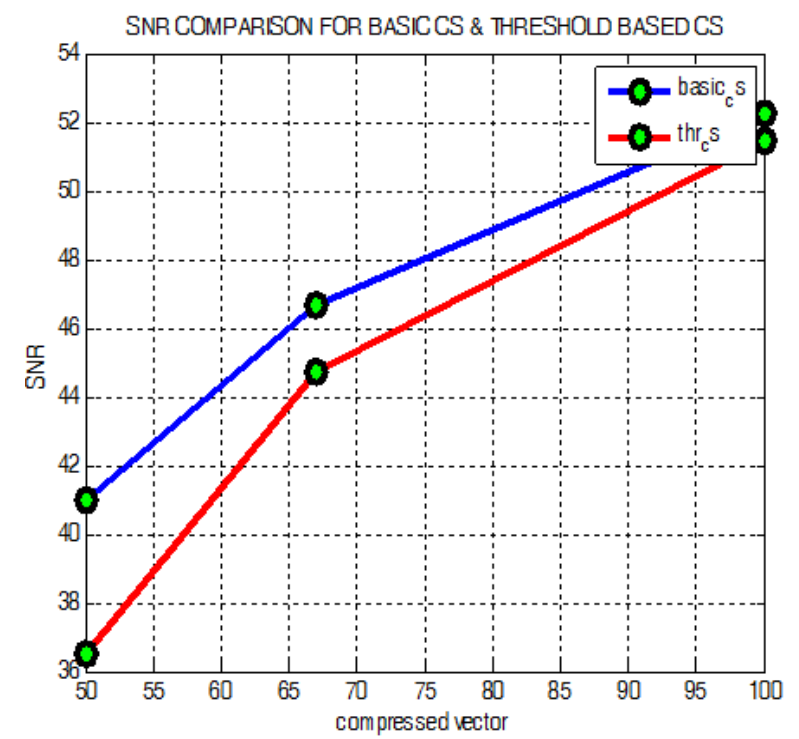

Fig. 1. SNR (in DB) Comparison For Basic CS and Threshold Based CS For Highly Correlated Data

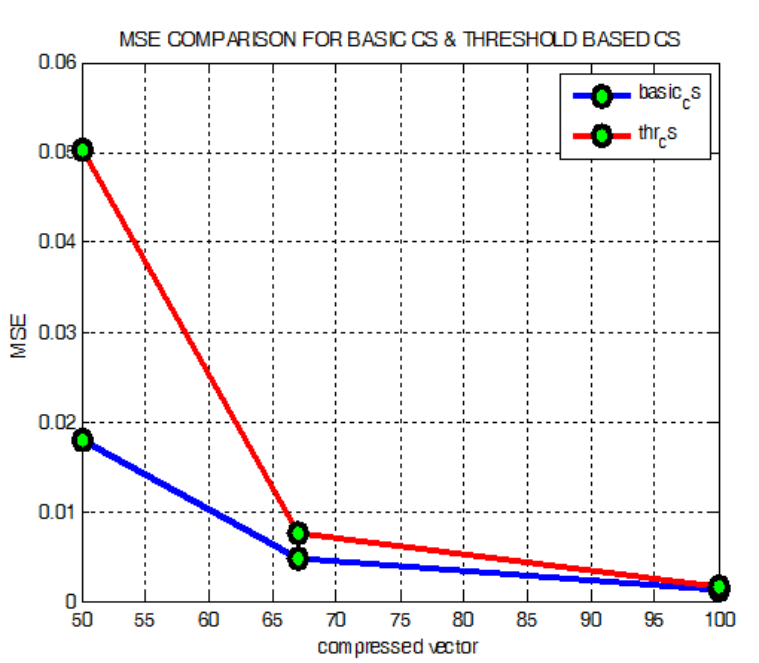

Fig. 2. MSE Comparison For Basic CS and Threshold Based Cs For Highly Correlated Data

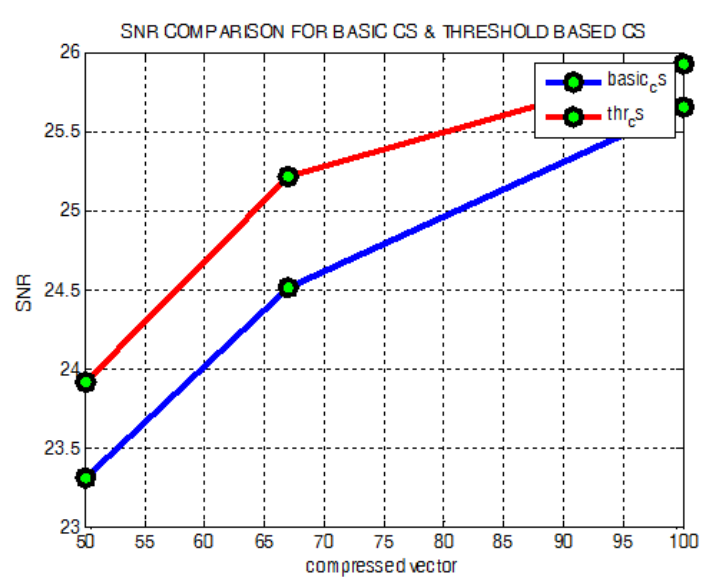

Fig. 3. SNR(in dB) Comparison For Basic CS and Threshold Based CS For Low Correlated Data

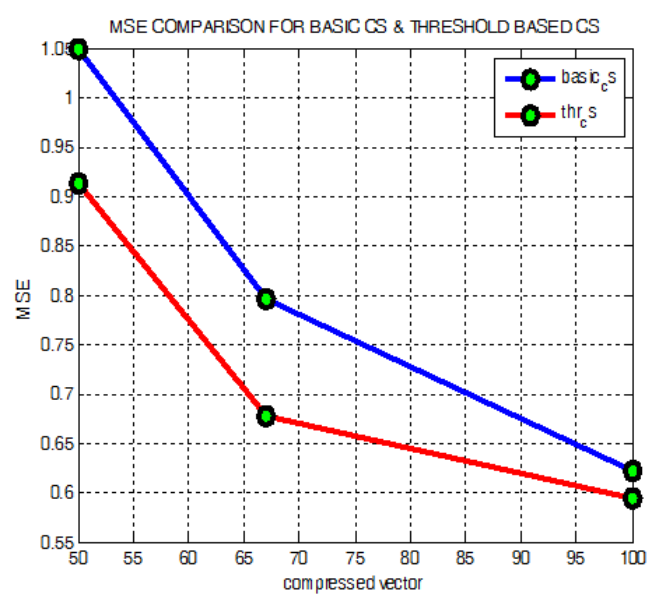

Fig. 4. MSE Comparison For Basic Cs and Threshold Based Cs For Low Correlated Data 


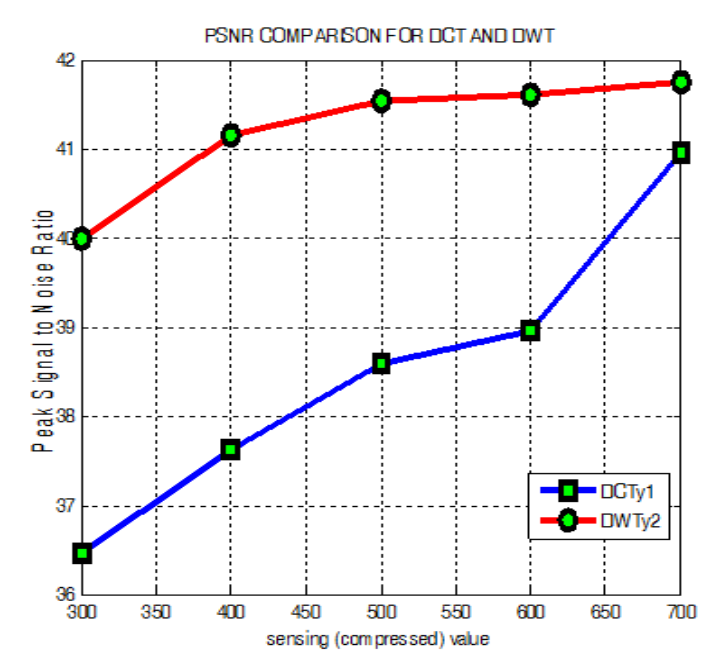

Fig. 5. Comparison of PSNR (in $\mathrm{dB}$ ) in DCT and DWT basis

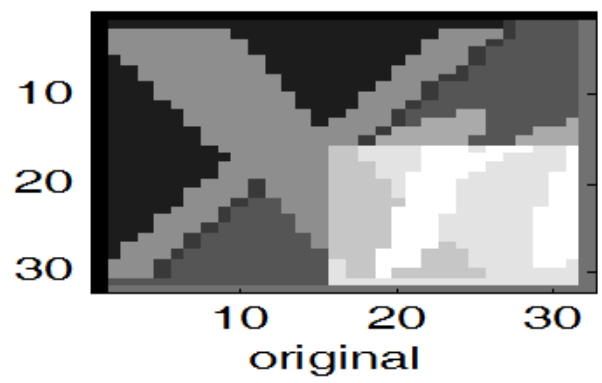

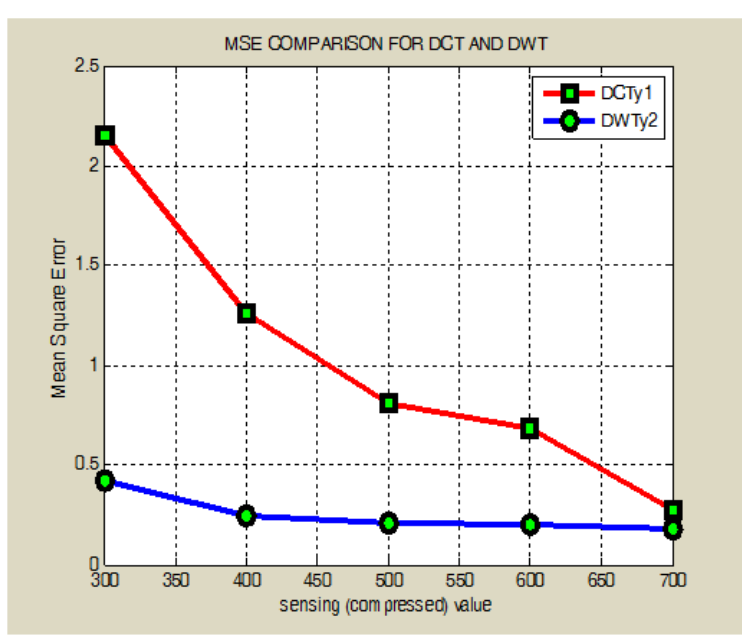

Fig. 6. Comparison of mse in DCT and DWT basis

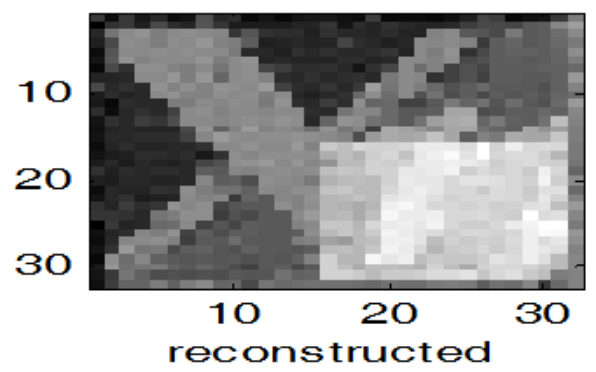

Fig. 7. Original input image and reconstructed image with DCT basis with Dimension of input image (N) $32 \times 32=1024$ pixels and Reconstructed image with $\mathrm{M}=360$ pixels
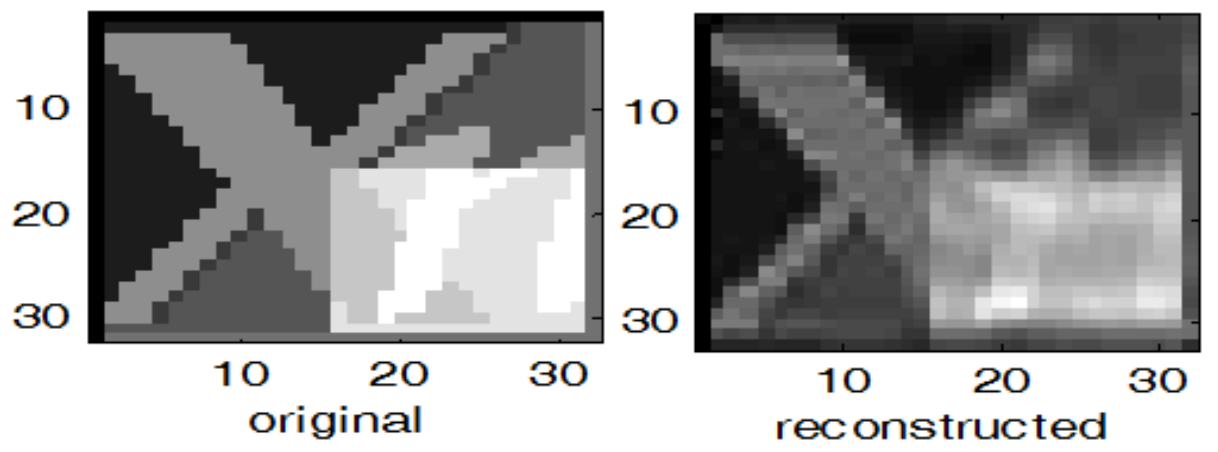

Fig. 8. Original input image and reconstructed image with DWT basis with Dimension of input image (N) $32 \times 32=1024$ pixels and Reconstructed image with $\mathrm{M}=360$ pixels

\section{Conclusion}

Simulation results show that, use of compressive sensing algorithm for data compression tremendously reduces the number of transmission bits as the SNR value is high and hence enhances the transmission and bandwidth efficiency in WSN. Results show that a suitable threshold value can increase the level of sparsity in transformed data when the input signal correlation is very low. The input data with low mean correlation coefficient gives better SNR and less MSE in case of one dimensional signal compared to highly correlated data when thresholding is incorporated. On the otherhand it is obvious from the result that basic CS without any threshold selection is much suitable for highly correlated data input. In case of image input signal comparison of two different basis is carried out. Results show that DWT is much suitable transform basis than DCT 
transform basis for sparse measurement generation in case of image input. In comparison with DCT, DWT is computationally simple and uses very less time which is expected in real time application. A high degree of compression ratio can be achieved with the help of proposed algorithms, which is shown in the result. The reconstruction result shows that even less than $50 \%$ of the data sample is sufficient to recover the original data and image, perhaps which is the most surprising result. Since the energy efficiency can be enhanced significantly using the proposed algorithm and hence the battery operated WSN lifetime can be prolonged.

\section{Acknowledgement}

The authors thank the BS Abdur Rahman University for providing the platform to carryout the research work.

\section{Funding Information}

The infrastructure facility of BS Abdur Rahman University is used for carrying out the research work.

\section{Author's Contributions}

Parnasree Chakraborty: The author has contributed to the experimental part and to the paper preparation.

C. Tharini: The author has contributed for verification of experimental result and for the paper preparation.

\section{Ethics}

The authors declare that the present article is a novel work and an outcome of original and exhaustive literature survey in the field of data compression in wireless sensor networks. The Authors also declare that there are no ethical issues that may arise after the publication of this manuscript.

\section{References}

Candès, E. and J. Romberg, 2005. 11-MAGIC: Recovery of sparse signals via convex programming. Caltech.

Candès, E.J. and J. Romberg, 2007. Sparsity and incoherence in compressive sampling. Inverse Problems, 23: 969-985.

DOI: $10.1088 / 0266-5611 / 23 / 3 / 008$

Candès, E.J. and M.B. Wakin, 2008. An introduction to compressive sampling. IEEE Signal Processing Magazine, 25: 21-30.

DOI: 10.1109/MSP.2007.914731
Candès, E.J. and T. Tao, 2006. Near optimal signal recovery from random projections: Universal encoding strategies. IEEE Trans. Info. Theory, 52: 5406-5425. DOI: 10.1109/TIT.2006.885507

Candès, E.J., J. Romberg and T. Tao, 2006. Stable signal recovery from incomplete and inaccurate measurements. Comm. Pure Appl. Math., 59: 1207-1223. DOI: 10.1002/cpa.20124

Karl, H. and A. Willing, 2007. Protocols and Architectures for Wireless Sensor Networks. 1st Edn., John Wiley \& Sons, ISBN-10: 0470519231, pp: 497.

Kolo, J.G. and S.A. Shanmugam, 2012. An adaptive lossless data compression scheme for wireless sensor networks. J. Sensors. DOI: $10.1155 / 2012 / 539638$

Li, Y., D. Qiao, Z. Xu, D. Xu and F. Miao et al., 2012. Energy-model-based optimal communication systems design for wireless sensor networks. Int. J. Distributed Sensor Networks.

Luke, H.D., 1999. The origins of the sampling theorem. IEEE Communications Magazine.

Luo, H., Y.C. Tong and G. Pottie, 2005. A two-stage DPCM scheme for wireless sensor networks. Proceedings of the International Conference on Acoustics, Speech and Signal Processing.

Mallat, S., 2008. A Wavelet Tour of Signal Processing: The Sparse Way, 3rd Edn., San Diego, CA: Academic Press, ISBN-10: 0080922023, pp: 832.

Marcelloni, F. and M. Vecchio, 2008. A simple algorithm for data compression in wireless sensor networks. IEEE Communications Letters.

Marcelloni, F., 2009. An efficient lossless compression algorithm for tiny nodes of monitoring wireless sensor networks. Computer J., 52: 969-987.

Medeiros, H.P. and M.C. Maciel, 2014. Lightweight data compression in wireless sensor networks using huffman coding. Int. J. Distributed Sensor Networks.

Nesterov, Y., 2004. Introductory lectures on convex optimization-A basic course. Clawer Academic Publishers, 87: 231-236. DOI: $10.1007 / 978-1-4419-8853-9$

Schurgers, C., O. Aberborne and M. Srivastava, 2001. Modulation scaling for energy-aware communication systems. In ISLPED, pp: 96-99.

Yang, G., M. Xiao and S. Zhang, 2013. Data aggregation scheme based on compressed sensing in wireless sensor network. J. Networks.

Yang, Z., Y. Yuan, J. He and W. Chen, 2005. Adaptive modulation scaling scheme for wireless sensor networks. IEICE. 